\title{
Preliminary Results from a Prospective Study using Limited Margin Radiotherapy in Pediatric and Young Adult High Grade Non-Rhabdomyosarcoma Soft Tissue Sarcoma
}

\author{
Matthew J. Krasin, M.D. ${ }^{1}$, Andrew M. Davidoff, M.D. ${ }^{2,6}$, Xiaoping Xiong, Ph.D. ${ }^{3}$, Shengjie Wu, \\ M.S. ${ }^{3}$, Chia-Ho Hua, Ph.D. ${ }^{1}$, Fariba Navid, M.D. ${ }^{4,5}$, Carlos Rodriguez-Galindo, M.D. ${ }^{4,5}$, \\ Bhaskar N. Rao, M.D. ${ }^{2}$, Kelly A. Hoth, B.S. ${ }^{1}$, Michael D. Neel, M.D. ${ }^{2}$, Thomas E. Merchant, \\ D.O., Ph.D. ${ }^{1}$, Larry E. Kun, M.D. ${ }^{1}$, and Sheri L. Spunt, M.D. 4,5 \\ ${ }^{1}$ Department of Radiological Sciences, St. Jude Children's Research Hospital, Memphis, TN, USA \\ ${ }^{2}$ Department of Surgery, St. Jude Children's Research Hospital, Memphis, TN, USA \\ ${ }^{3}$ Department of Biostatistics, St. Jude Children's Research Hospital, Memphis, TN, USA \\ ${ }^{4}$ Department of Oncology, St. Jude Children's Research Hospital, Memphis, TN, USA \\ ${ }^{5}$ Department of Pediatrics, University of Tennessee College of Medicine, Memphis, TN, USA \\ ${ }^{6}$ Department of Surgery, University of Tennessee College of Medicine, Memphis, TN, USA
}

\section{Abstract}

Purpose-To demonstrate the safety and efficacy of limited margin radiotherapy in the local control of pediatric and young adult patients with high grade non-rhabdomyosarcoma soft tissue sarcoma (NRSTS).

\begin{abstract}
Methods and Materials-Pediatric patients with high-grade NRSTS requiring radiation were treated on an IRB approved prospective institutional study of conformal / intensity modulated / interstitial brachytherapy using a $2 \mathrm{~cm}$ anatomically constrained margin.

Results-Thirty-two patients (median age 15.3 years, range 2-22 years) received adjuvant (27 patients) or definitive (5 patients) irradiation. With a median follow-up of 32 months, the 3 -year cumulative incidence of local failure was $3.7 \%$ for patients irradiated after surgical resection. In total four patients experienced local failure; the mean dose to the volume of recurrence was $\geq 97 \%$ of the prescribed dose.
\end{abstract}

Conclusions-Delivery of limited margin radiotherapy using external beam or brachytherapy provides a high rate of local tumor control without marginal failure. Further follow-up is required to determine if normal tissue effects are minimized using this approach.

(C) 2009 Elsevier Inc. All rights reserved.

Corresponding author: Matthew J. Krasin, M.D. St. Jude Children's Research Hospital, 262 Danny Thomas Place, Memphis, TN 38105 , Phone: (901) 595-3226, Fax: (901) 595-3113, matthew.krasin@ @stjude.org.

Publisher's Disclaimer: This is a PDF file of an unedited manuscript that has been accepted for publication. As a service to our customers we are providing this early version of the manuscript. The manuscript will undergo copyediting, typesetting, and review of the resulting proof before it is published in its final citable form. Please note that during the production process errors may be discovered which could affect the content, and all legal disclaimers that apply to the journal pertain.

Presentation: American Society for Therapeutic Radiology and Oncology’s 2006 Annual Meeting, Philadelphia, PA

Conflicts of Interest Notification

No conflicts of interest exist. 


\section{Keywords}

Soft-tissue sarcoma; Conformal; IMRT; Margin; Radiation

\section{Introduction}

The management of pediatric, adolescent and young adult patients (AYA) diagnosed with nonrhabdomyosarcoma soft tissue sarcoma has evolved from the adult paradigms of management (1-3). In current practice, the primary site of disease is managed with surgical resection, and radiation therapy is incorporated based on initial resectability, tumor grade and margin status (4-6). The indications for systemic chemotherapy are controversial; patients with unresectable disease and those with high-grade primary tumors $>5 \mathrm{~cm}$ are often treated pre- or postoperatively $(7,8)$. When radiation therapy is indicated, pediatric patients are approached differently because of the desire to avoid late effects including impaired growth and development and potentially devastating life-long complications (9). Treatment guidelines in clinical trials for adult soft tissue sarcomas, particularly those of the extremity, have recommended target volume margins as large as $5 \mathrm{~cm}$ beyond the primary tumor or resection bed. They incorporate a large volume of normal tissue and often irradiate the surgical incision $(10,11)$. When delivered in children, the adult approach using large treatment volumes and opposed tangential treatment beams places adjacent tissues at risk for late effects that include reduced bone growth, loss of joint function and soft tissue and muscle fibrosis (12-15). This has resulted in pediatric clinical trials that have lacked specific radiation treatment guidelines or avoided radiation resulting in outcome data that do not provide clear guidance as to the benefit or detriment of radiation therapy (16).

To address these issues, we designed a prospective trial to test the efficacy of focal limited margin radiation therapy delivered with conformal radiation, intensity modulated radiation or an interstitial brachytherapy approach. We report the initial results of this study for patients with high grade non-rhabdomyosarcoma soft tissue sarcoma, describing local disease control, patterns of failure and toxicity.

\section{Methods and Materials}

Pediatric and AYA patients with high grade non-rhabdomyosarcoma soft tissue sarcoma (NRSTS) requiring radiation were treated on an IRB approved prospective phase II institutional study of limited margin radiation therapy. Eligibility included patients $<25$ years of age that required radiation therapy for management of their primary site of disease. Any primary site of involvement was allowed, and patients with metastatic disease were included. The primary objective of this clinical trial was to define the local tumor control rate for the NRSTS patient population with this radiotherapeutic approach. Secondary objectives, discussed in part in this manuscript, sought to define the early and late toxicity profile of this limited margin treatment approach related to the musculoskeletal system. Patients enrolled on this clinical trial were treated with radiation therapy, primarily in conjunction with surgical resection of the primary site of disease. Selection of patients necessitating preoperative, postoperative or definitive radiation was based on the presence of high grade sarcoma, positive microscopic margins and/ or limited resectability (in cases receiving pre-operative or definitive irradiation). Degree of surgical resection was classified based on the description by Enneking and the pathologic margin status (17). Stage was assigned based on AJCC version 6 (18).

Treatment consisted of ICRU 50 target volumes generated based on CT and MR treatment planning datasets with MR data sets (typically obtained in the treatment position) co-registered to the CT to fully define target volumes (19). The gross tumor volume (GTV) was the post- 
operative tumor bed or gross tumor constrained by barriers to tumor spread (bone, fascial planes) defined by the treating physician. A $2.0 \mathrm{~cm}$ clinical target volume (CTV) margin was added to the GTV, also anatomically constrained by barriers to tumor spread. The surgical incision was not targeted specifically. A site and patient specific planning target volume (PTV) was added that ranged from $0.4 \mathrm{~cm}$ to $1.0 \mathrm{~cm}$. There was no PTV for brachytherapy cases. Radiation delivery was accomplished with conformal radiation therapy, intensity modulated radiation therapy, interstitial brachytherapy or a combination of these modalities. External beam radiation was delivered with 6 or $15 \mathrm{MV}$ photons or megavoltage electrons while brachytherapy was delivered with low dose rate interstitial Iridium-192 seeds arranged in catheters and spaced $1.0 \mathrm{~cm}$ apart to deliver a homogenous dose. Specifics of external beam delivery techniques have been reported (20). Protocol specified total doses of radiation therapy were 55.8-63 Gy for postoperative radiation, 45-50.4 Gy for preoperative radiation and 70.2 Gy for definitive radiation, all delivered at $1.8 \mathrm{~Gy}$ per fraction. When interstitial brachytherapy was used alone, $45 \mathrm{~Gy}$ was prescribed.

Deviations from these dosing guidelines were made based on protection of adjacent normal tissues. Systemic therapy was at the discretion of the treating pediatric oncologist. In general, it was based on initial resectability and high risk features [large tumor size $(>5 \mathrm{~cm})$ and high histologic grade].

Follow-up for patients included a history, physical exam of the primary site of disease, MR imaging of the primary site, $\mathrm{CT}$ of the chest and other involved site imaging every 3 months for 2 years and then every 6 months until 5 years from protocol enrollment. Positron emission tomography (PET) with ${ }^{18} \mathrm{~F}$-fluorodeoxyglucose (FDG) was obtained at baseline, 6 months, 1 year and 2 years of follow-up. Toxicity was assessed weekly during radiation and at each follow-up visit according to the Common Toxicity Criteria version 2.0 (21).

Treatment failure was classified as local if the recurrence was within the PTV. Adjacent or marginal failures were also considered local for the purpose of defining the cumulative incidence. The cumulative incidence (CIN) of local failure was estimated by Gray's method and was defined as time to local failure with other events considered competing (22). The event free survival (EFS) was defined as the time to any event including local failure, distant failure, secondary malignancy or death prompting removal of the patient from the study. The KaplanMeier method was used to estimate event free survival (23).

\section{Results \\ Patient Population}

Thirty-two patients with high grade NRSTS were enrolled on this study from March 2003 through June 2007. The median age at the time of study enrollment was 15.3 years (range, 2.522.9). Fourteen patients were female, and 18 were male. The median follow-up of all patients remaining on study at the time of analysis was 32 months (range, 14-66 months). Tumor and treatment characteristics are shown in Table 1. Twenty-five patients received external beam radiation therapy, eight in conjunction with brachytherapy. Seven patient received brachytherapy without external beam radiation in conjunction with surgical resection. In some patient cases adjacent normal tissue tolerances limited delivery of the protocol specified prescription dose. The median cumulative dose delivered for patients receiving external beam radiation was 60 Gy (range, 41.4-70.4 Gy) in the post-operative setting, 45 Gy (range, 45$50.4 \mathrm{~Gy}$ ) pre-operative and 50.4 Gy (range, 23.4-70.2 Gy) definitively. The median cumulative dose of post-operative brachytherapy delivered with external beam radiation therapy was 17.1 Gy (range, 13.8-20.0 Gy). Forty-five Gy was delivered in all patients treated with postoperative brachytherapy alone. 


\section{Local Failure}

The 3-year cumulative incidence of local failure for the entire cohort was $12.5 \%$ (standard error (SE), 6\%). Patients undergoing a marginal or complete resection with radiation therapy had a 3-year cumulative incidence of local failure of $3.7 \%$ (SE, 3.7\%), while those patients receiving definitive radiation following biopsy had a 1-year cumulative incidence of local failure of $40 \%$ (SE, $28 \%, \mathrm{p}=0.0007$, figure 1 ). There were no significant differences in the incidence of local failure based on patients' primary tumor T-stage $(\mathrm{T} 1[\leq 5 \mathrm{~cm}]$ vs. T2 [>5cm], $\mathrm{p}=0.60)$, overall stage (IIB vs. III vs. IV, $\mathrm{p}=0.40$ ), or tumor location (extremity vs. head and neck vs. trunk, $\mathrm{p}=0.37$ ). There also were no local failures in patients that had clear surgical margins, while the cumulative incidence of local failure for patients with positive surgical margins was $6.7 \%$ at 3 years $(\mathrm{p}=0.37)$. In patients undergoing resection, there was no difference in local control based on the addition of systemic therapy $(\mathrm{p}=0.27)$.

\section{Event free survival}

Event free survival for the entire cohort of 32 patients was $55.0 \%$ at 3 years. Patients that underwent resection fared significantly better than patients treated with definitive radiation having an EFS of $74.1 \%$ vs. $20.0 \%$ at 1 year and $65.2 \%$ vs. $0 \%$ at 3 years, respectively. The presence of metastatic disease significantly reduced EFS at 1-year $(28.6 \%$ vs. $76.0 \%$, $\mathrm{p}<0.0001)$. Delivery of systemic therapy in the context of resection resulted in no significant difference in EFS (3-year EFS, 68.1\%) compared to management without systemic therapy (3year EFS, $58.3 \%$, $\mathrm{p}=0.44$ ), although patients were not selected for systemic therapy uniformly.

\section{Patterns of treatment failure}

Four patients experienced a local treatment failure, three of which had only undergone biopsy and definitive radiation as local management. The remaining local failure occurred at the primary site in the wrist of a child with epitheloid sarcoma following marginal resection, 15 Gy interstitial brachytherapy and $45 \mathrm{~Gy}$ external beam radiation therapy. All local recurrences were within the high dose region of the patient's treatment plan based on co-registration of the recurrence imaging with the patients' radiation dosimetry. In all cases the mean dose in the volume of recurrence was $97 \%$ of the prescribed dose or greater. Ten patients had metastatic failure as their first recurrence, one of which was concurrent with a local failure. Metastatic failures were located in the lung/pleura (six) and regional lymph node, brain, bone and peritoneum (one each). The median times to local and distant failure were 9.9 months and 5.0 months, respectively.

\section{Toxicity}

In general, radiation therapy was well tolerated. The maximal toxicity noted for each patient by category is shown in Table 2 . Two grade 4 toxicities were seen. In one patient skin necrosis occurred 7 months following treatment with 15 Gy interstitial brachytherapy and 45 Gy external beam radiation therapy. In the second patient a metatarsal fracture occurred 11 months following 63 Gy external beam radiation therapy delivered to the dorsum of the foot.

\section{Discussion}

We described the outcome for a prospectively treated well-defined cohort of patients managed with limited margin radiation therapy and either surgical resection or biopsy. Important to this study was the use of an anatomically constrained $2.0 \mathrm{~cm}$ clinical target volume margin designed to limit adjacent normal tissue dose. Despite the presence of reported adverse clinical factors including less than wide local surgical resections as well as primary tumor sites including the trunk and head and neck, the cumulative incidence of local failure in our patients undergoing resection was only $3.7 \%(24,25)$. Patterns of treatment failure indicate that sites of recurrence 
were within the high dose treatment volume without marginal failure suggesting that in the pediatric population a $2.0 \mathrm{~cm}$ CTV margin is acceptable. It is important to note that on our study treatment planning was done with both $\mathrm{CT}$ and co-registered MR data sets typically obtained in the treatment position to fully define target volumes. This limited margin in the context of modern conformal or intensity modulated radiotherapy techniques reduces extratarget high dose volumes and may reduce long term treatment related effects. These findings are similar to the recently published adult experience of intensity modulated radiation in the extremity with a local control rate of $94 \%$, employing similar radiation doses but larger treatment margins (26).

Despite radiation therapy treatment plans conformed to the primary tumor, $47 \%$ of patients were noted to have grade II or greater late soft tissue fibrosis, and $31 \%$ of patients were found to have moderate stiffness or reduction in joint range of motion related to radiation therapy. The further evolution and relation of these toxicities to the patients' dosimetry and clinical factors such as age at treatment will be explored in subsequent publications. Our experience with other radiation related late effects suggests that both clinical and dosimetric factors can be incorporated into a descriptive model providing a quantitative estimate of the toxicity (14). It will be important to identify whether these effects are related to high doses of radiation therapy directly adjacent to the clinical target volume or within the planning target volume or whether lower doses outside of the intended treatment region are responsible for these side effects.

Only the degree of surgical resection (biopsy vs. resection) was significant for an increased incidence of local failure. In the 12 patients undergoing a wide local excision achieving clear surgical margins, six had tumor less than $5.0 \mathrm{~cm}$ in the greatest dimension. Based on retrospective data in adults, this group of pediatric patients who have undergone a wide resection may be candidates for observation alone in the context of a clinical trial (as is being done in Children's Oncology Group trial ARST0332) (27-30). Patients with primary disease involving the trunk or head and neck did not experience an adverse local outcome despite the historic adverse prognosis that these features would predict $(24,25)$. It is also interesting to note that in patients with metastatic disease there was no difference in local disease control. Despite these factors, the consistent targeting approach employed in this study resulted in high rates of local disease control.

\section{Conclusions}

A modern radiotherapeutic targeting and treatment approach yields excellent local disease control rates in pediatric and AYA patients diagnosed with non-rhabdomyosarcoma soft tissue sarcoma. This limited margin approach provides a benchmark for disease control and comparisons with future approaches including radiation dose reduction, volume reduction or application of new radiation technologies.

\section{Acknowledgments}

Support: American Lebanese Syrian Associated Charities (ALSAC), Lance Armstrong Foundation

\section{References}

1. Lindberg RD, Martin RG, Romsdahl MM, Barkley HT Jr. Conservative surgery and postoperative radiotherapy in 300 adults with soft-tissue sarcomas. Cancer 1981;47(10):2391-2397. [PubMed: 7272893]

2. Yang JC, Chang AE, Baker AR, et al. Randomized prospective study of the benefit of adjuvant radiation therapy in the treatment of soft tissue sarcomas of the extremity. J Clin Oncol 1998;16(1):197-203. [PubMed: 9440743] 
3. Pisters PW, Harrison LB, Leung DH, et al. Long-term results of a prospective randomized trial of adjuvant brachytherapy in soft tissue sarcoma. J Clin Oncol 1996;14(3):859-868. [PubMed: 8622034]

4. Fleming JB, Berman RS, Cheng SC, et al. Long-term outcome of patients with American Joint Committee on Cancer stage IIB extremity soft tissue sarcomas. J Clin Oncol 1999;17(9):2772-2780. [PubMed: 10561352]

5. LePechoux C, Le Deley M-C, Delaloge S, et al. Postoperative radiotherapy in the management of adult soft tissue sarcoma of the extremities: Results with two different total dose, fractionation, and overall treatment time schedules. Int J Radiat Oncol Biol Phys 1999;44:879-886. [PubMed: 10386645]

6. Spunt SL, Poquette CA, Hurt YS, et al. Prognostic factors for children and adolescents with surgically resected nonrhabdomyosarcoma soft tissue sarcoma: An analysis of 121 patients treated at St Jude Children's Research Hospital. J Clin Oncol 1999;17(12):3697-3705. [PubMed: 10577841]

7. Bramwell VH. Adjuvant chemotherapy for adult soft tissue sarcoma: Is there a standard of care. J Clin Oncol 2001;19(5):1235-1237. [PubMed: 11230463]

8. Kraybill WG, Harris J, Spiro IJ, et al. Phase II study of neoadjuvant chemotherapy and radiation therapy in the management of high-risk, high-grade, soft tissue sarcomas of the extremities and body wall: Radiation Therapy Oncology Group Trial 9514. J Clin Oncol 2006;24(4):619-625. [PubMed: 16446334]

9. Merchant TE. Conformal Therapy for Pediatric Sarcomas. Semin Radiat Oncol 1997;7(3):236-245. [PubMed: 10717219]

10. Mundt AJ, Awan A, Sibley GS, et al. Conservative surgery and adjuvant radiation therapy in the management of adult soft tissue sarcoma of the extremities: Clinical and radiobiological results. Int J Radiat Oncol Biol Phys 1995;32(4):977-985. [PubMed: 7607972]

11. Kim YB, Shin KH, Seong J, et al. Clinical significance of margin status in postoperative radiotherapy for extremity and truncal soft-tissue sarcoma. Int J Radiat Oncol Biol Phys 2008;70(1):139-144. [PubMed: 17919843]

12. Silber JH, Littman PS, Meadows AT. Stature loss following skeletal irradiation for childhood cancer. J Clin Oncol 1990;8(2):304-312. [PubMed: 2299373]

13. Denys D, Kaste SC, Kun LE, et al. The effects of radiation on craniofacial skeletal growth: A quantitative study. Int J Pediatric Oto Rhino Laryngology 1998;45:7-13.

14. Krasin MJ, Xiaoping X, Shengjie W, et al. The effects of external beam irradiation on the growth of flat bones in children: Modeling a dose-volume effect. Int J Radiat Oncol Biol Phys 2005;62(5): 1458-1462. [PubMed: 16029808]

15. Goldstein M, Maxymiw WG, Cummings BJ, Wood RE. The effects of antitumor irradiation on mandibular opening and mobility: A prospective study of 58 patients. Oral Surg Oral Med Oral Pathol Oral Radiol Endod 1999;88(3):365-373. [PubMed: 10503870]

16. Pratt CB, Pappo AS, Gieser P, et al. Role of adjuvant chemotherapy in the treatment of surgically resected pediatric nonrhabdomyosarcomatous soft tissue sarcomas: A Pediatric Oncology Group Study. J Clin Oncol 1999;17(4):1219. [PubMed: 10561182]

17. Enneking WF. A system of staging musculoskeletal neoplasms. Clin Orthop Relat Res 1986;(204): 9-24. [PubMed: 3456859]

18. American Joint Committee on Cancer. AJCC cancer staging manual. 6th ed.. New York: Springer; 2002. p. 193-197.

19. ICRU. Prescribing, recording, and reporting photon beam therapy from the International Commission of Radiation Units and Measurements (ICRU). 1993 Report No.: 50.

20. Hua C, Gray JM, Merchant TE, Kun LE, Krasin MJ. Treatment planning and delivery of external beam radiotherapy for pediatric sarcoma: The St. Jude Children's Research Hospital experience. Int J Radiat Oncol Biol Phys 2008;70(5):1598-1606. [PubMed: 18234441]

21. National Institute of Health. Common Toxicity Criteria, version 2.0. Available at: http://ctep.info.nih.gov/reporting/ctc.html

22. Gray RJ. A class of K-sample tests for comparing the cumulative incidence of a competing risk. Ann Stat 1988;16:1141-1154.

23. Kaplan EL, Meier P. Nonparametric estimation from incomplete observations. J Am Stat Assoc 1958;53:457. 
24. Ferrari A, Bisogno G, Alaggio R, et al. Synovial sarcoma of children and adolescents: The prognostic role of axial sites. Eur J Cancer 2008;44(9):1202-1209. [PubMed: 18440800]

25. Harb WJ, Luna MA, Patel SR, et al. Survival in patients with synovial sarcoma of the head and neck: Association with tumor location, size, and extension. Head Neck 2007;29(8):731-740. [PubMed: 17274049]

26. Alektiar KM, Brennan MF, Healey JH, Singer S. Impact of intensity modulated radiation therapy on local control in primary soft-tissue sarcoma of the extremity. J Clin Oncol 2008;26(20):3440-3444. [PubMed: 18612160]

27. Cahlon O, Spierer M, Brennan MF, Singer S, Alektiar KM. Long-term outcomes in extremity soft tissue sarcoma after a pathologically negative re-resection and without radiotherapy. Cancer 2008;112(12):2774-2779. [PubMed: 18429001]

28. Baldini EH, Goldberg J, Jenner C, et al. Long-term outcomes after function-sparing surgery without radiotherapy for soft tissue sarcoma of the extremities and trunk. J Clin Oncol 1999;17(10):32523259. [PubMed: 10506627]

29. Rydholm A, Gustafson P, Rooser B, et al. Limb-sparing surgery without radiotherapy based on anatomic location of soft tissue sarcoma. J Clin Oncol 1991;9(10):1757-1765. [PubMed: 1919628]

30. Fabrizio PL, Stafford SL, Pritchard DJ. Extremity soft-tissue sarcomas selectively treated with surgery alone. Int J Radiat Oncol Biol Phys 2000;48(1):227-232. [PubMed: 10924993] 


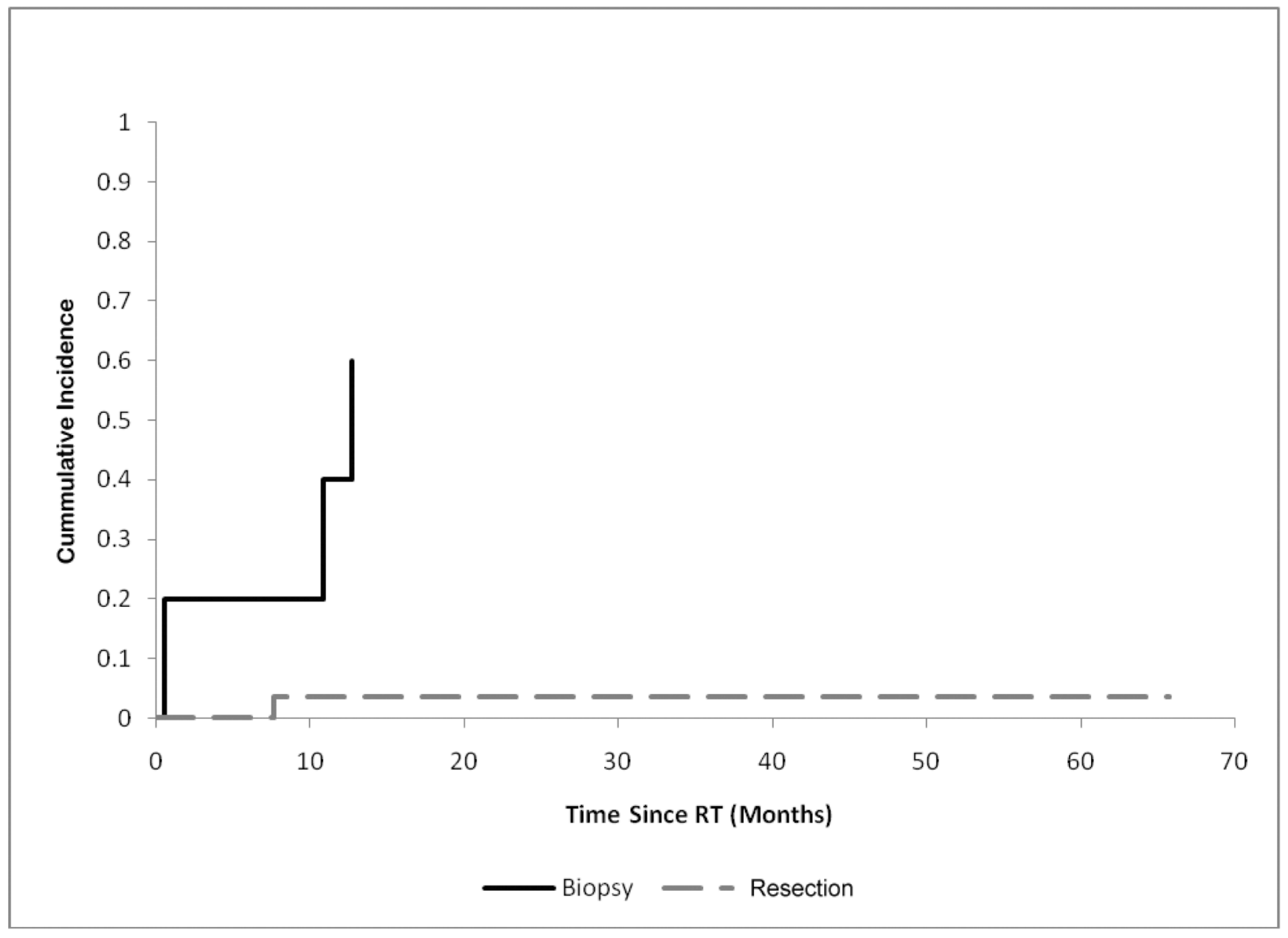

Figure 1.

Cumulative incidence of local failure by degree of resection 
Table 1

Patient Characteristics

\begin{tabular}{|c|c|c|}
\hline & Number & Percent $(\%)$ \\
\hline Histology & \multirow[b]{2}{*}{6} & \multirow[b]{2}{*}{$19 \%$} \\
\hline Synovial cell sarcoma & & \\
\hline Malignant peripheral nerve sheath tumor & 6 & $19 \%$ \\
\hline Epitheloid sarcoma & 4 & $12 \%$ \\
\hline Mesenchymal chondrosarcoma & 3 & $9 \%$ \\
\hline Other high grade sarcoma & 13 & $41 \%$ \\
\hline Tumor Depth & \multirow[b]{2}{*}{1} & \multirow[b]{2}{*}{$3 \%$} \\
\hline Superficial & & \\
\hline Deep & 31 & $97 \%$ \\
\hline Tumor Size & \multirow[b]{2}{*}{12} & \multirow[b]{2}{*}{$38 \%$} \\
\hline$\leq 5 \mathrm{~cm}$ & & \\
\hline$>5 \mathrm{~cm}$ to $\leq 10 \mathrm{~cm}$ & 11 & $34 \%$ \\
\hline$>10 \mathrm{~cm}$ & 9 & $28 \%$ \\
\hline T Stage & \multirow[b]{2}{*}{1} & \multirow[b]{2}{*}{$3 \%$} \\
\hline $\mathrm{T} 1 \mathrm{~A}$ & & \\
\hline $\mathrm{T} 1 \mathrm{~B}$ & 11 & $34 \%$ \\
\hline $\mathrm{T} 2 \mathrm{~B}$ & 20 & $63 \%$ \\
\hline AJCC Overall Stage & \multirow[b]{2}{*}{12} & \multirow[b]{2}{*}{$37 \%$} \\
\hline IIB & & \\
\hline III & 13 & $41 \%$ \\
\hline IV & 7 & $22 \%$ \\
\hline Tumor Location & \multirow[b]{2}{*}{17} & \multirow[b]{2}{*}{$53 \%$} \\
\hline Extremity & & \\
\hline Head \& neck & 3 & $9 \%$ \\
\hline Trunk & 12 & $38 \%$ \\
\hline Degree of Resection & \multirow[b]{2}{*}{5} & \multirow[b]{2}{*}{$16 \%$} \\
\hline Biopsy & & \\
\hline Intralesional excision & 1 & $3 \%$ \\
\hline Marginal & 14 & $44 \%$ \\
\hline Wide local excision & 12 & $37 \%$ \\
\hline Chemotherapy & \multirow[b]{2}{*}{12} & \multirow[b]{2}{*}{$38 \%$} \\
\hline No & & \\
\hline Yes & 20 & $62 \%$ \\
\hline Doxorubicin / Ifosfamide based & 11 & $55 \%$ \\
\hline Other & 9 & $45 \%$ \\
\hline Radiation Therapy Regimen & \multirow[b]{2}{*}{24} & \multirow[b]{2}{*}{$75 \%$} \\
\hline Post-operative radiation & & \\
\hline
\end{tabular}




\begin{tabular}{|r|c|c|}
\hline & Number & Percent $(\%)$ \\
\hline Pre-operative radiation & 3 & $9 \%$ \\
\hline Definitive radiation & 5 & $16 \%$ \\
\hline
\end{tabular}




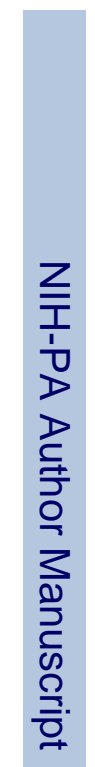

Krasin et al.

Page 11

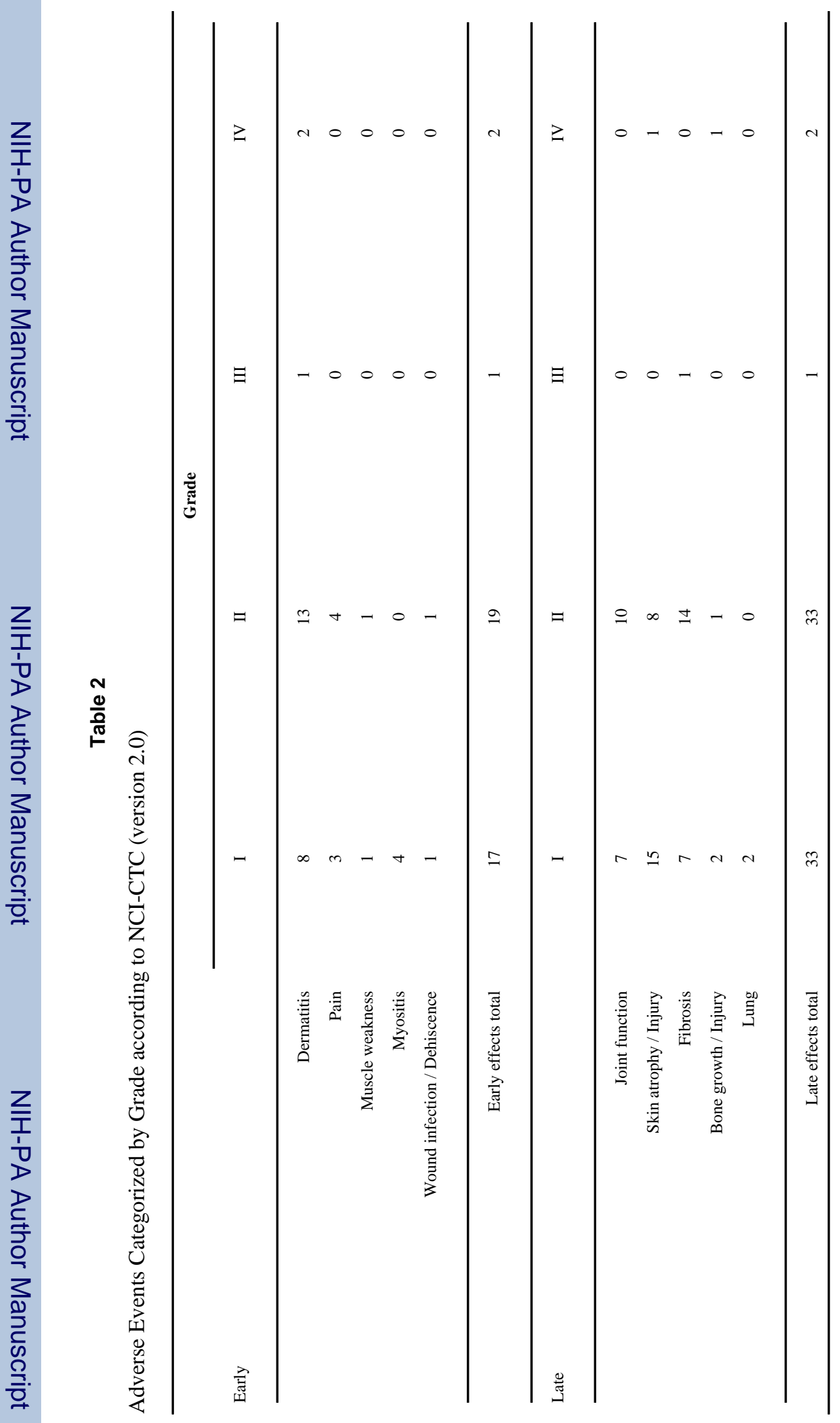

Int J Radiat Oncol Biol Phys. Author manuscript; available in PMC 2011 March 1. 